\title{
Differential inverse inelastic mean free path and differential surface excitation probability retrieval from electron energy loss spectra
}

\author{
Viktor P. Afanas'ev', Alexander S. Gryazev ${ }^{\mathrm{a}}$, Dmitry S. Efremenko ${ }^{\mathrm{b}}$, Pavel S. Kaplya ${ }^{\mathrm{a}, *}$ \\ ${ }^{a}$ Department of General Physics and Nuclear Fusion, National Research University "Moscow Power Engineering Institute", Krasnokazarmennaya, 14, Moscow \\ 111250, Russia \\ ${ }^{b}$ Deutsches Zentrum für Luft- und Raumfahrt (DLR), Institut für Methodik der Fernerkundung (IMF), 82234 Oberpfaffenhofen, Germany
}

\begin{abstract}
Quantitative interpretation of the electron spectroscopy data requires the information on differential inverse inelastic mean free paths (DIIMFP) and differential surface excitation probabilities (DSEP). In this paper, we test an algorithm of extracting DIIMFP and DSEP from reflected electron energy loss spectra (REELS) and photo-electron spectra (PES) in which the desired functions are parametrized on the base of linear respond theory. Unknown parameters are found by using the fitting procedure. To account for surface excitations, the investigated samples are considered as multi-layer systems. Simulations of REELS and PES are performed by making use of the partial intensity approach. The partial intensities for the reflection function and the photo-electron density flux are computed on the base of the invariant imbedding method. Extracted DIIMFPs and DSEPs are compared with those obtained by other authors. Finally, REELS and PES spectra for $\mathrm{Be}, \mathrm{Mg}, \mathrm{Al}, \mathrm{Si}, \mathrm{Nb}$ and $\mathrm{W}$ are computed using the retrieved DIIMFPs and DSEPs, and compared with the experimental spectra. All comparisons show good agreement.
\end{abstract}

Keywords: DIIMFP extraction, REELS deconvolution, PES, invariant imbedding, XPS, electron cross-section PACS: $34.80 . \mathrm{Bm}, 34.80 . \mathrm{Dp}$, 81.70.Jb, 73.20.Mf, 29.30.Dn

\section{Introduction}

Knowledge of the inelastic scattering parameters of solids is important for quantitative understanding of the energy loss process. The differential inverse inelastic mean free path (DIIMFP) and the differential surface excitation probability (DSEP) give the distribution of energy losses per unit path length in an individual inelastic collision in bulk and surface layers of solids, respectively. They are the main quantities characterizing inelastic scattering in solids. However, in practice, only integral quantities such as the inelastic mean free path (IMFP) and the electron stopping power are available in spectroscopic databases (e.g. NIST database by Powell and Jablonski [1]). Studies involving linear response theory [2] can predict only the general shape of the DIIMFP, while using more sophisticated approaches (e.g. based on density functional theory [3]) is complicated for real atomic structures. Bearing that in mind, it seems to be more feasible to extract information on the DIIMFP and DSEP from experimental optical data [4] or REELS spectra rather than to compute them from basic physical principles.

A convenient numerical framework for the REELS spectra analysis is the partial intensity approach [5, 6], in which a REELS spectrum is given by the weighted sum of multiple cross convolutions of DIIMFP and DSEP functions. The corresponding weighting factors are referred to as "partial intensities". To obtain DIIMFP and DSEP from REELS, the latter

\footnotetext{
* Corresponding author

Email address: pavel@kaplya.com (Pavel S. Kaplya)
}

has to be deconvolved to filter out multiple scattering effects. Several techniques have been proposed to retrieve DIIMFP and DSEP from REELS spectra employing the partial intensity approach. A direct numerical inversion scheme was proposed by Tougaard and Chorkendorff [7], and Tougaard and Kraaer [8] to extract DIIMFP from a REELS spectrum. The $P_{1}$-approximation was used to compute the partial intensities. The main drawback of this scheme is that it does not take into account surface excitations. As a result, the extracted "effective" DIIMFP (being some kind of mixture of the actual DIIMFP, DSEP and their cross-convolution) can have negative values in the region corresponded to the cross-convolution of DIIMFP and DSEP. Further, this approach was extended by Werner (e.g., see [9, 10]) to two-layer systems. There, DIIMFP and DSEP are retrieved from a pair of REELS spectra by reversing the bi-variate power series in Fourier space. A similar technique has been proposed by Afanas'ev et al. [11] which employs the REELS expansion through partial intensities only in the original space.

Note, that all direct numerical inversion schemes above are severely ill-posed, i.e. the noise in the spectrum results in physically irrelevant peaks in the shapes of extracted DIIMFP and DSEP. To regularize the inversion, Werner [12] proposed to fit extracted functions to the Drude-Lindhard model and in this way to get a physically-consistent result.

The intent of this paper is to test a method for DIIMFP and DSEP retrieval from REELS and PES spectra in which the desired functions are parametrized on the base of linear respond theory. Unknown parameters of the model are found by means of the fitting procedure. An important part of our retrieval algo- 
rithm is the fast yet accurate method for partial intensity computations. It employs ideas of Waterman [13] and Flatau and Stephens [14], and relies on the numerical solution of the invariant imbedding equations for scattered electrons. The forward simulations for REELS and PES are performed in the same framework, so that the DIIMFP functions extracted from REELS and PES can be cross-validated.

The rest of the paper is organized as follows. In Section 2, we briefly review the partial intensities approach and describe a technique for computing partial intensities using the invariant imbedding method. Section 3 provides basic relations for reflection and transmission functions in the case of multi-layer systems. A description of the retrieval algorithm set-up follows in Section 4. Here, DIIMFP and DSEP functions are extracted from REELS and PES spectra for a set of materials (Be, $\mathrm{Mg}$, $\mathrm{Al}, \mathrm{Si}, \mathrm{Nb}$ and $\mathrm{W}$ ). Section 5 summarizes the present work and outlines future tasks.

\section{Evaluation of partial intensities for single layers}

In this section we consider a single layer illuminated by the electron beam or the X-ray irradiation. Expanding the reflection function $R\left(\tau, \Delta, \mu_{0}, \mu, \varphi\right)$ of electrons into a Fourier cosine series gives

$$
R\left(\tau, \Delta, \mu_{0}, \mu, \varphi\right)=\sum_{m=0}^{\infty}\left(2-\delta_{m 0}\right) R^{m}\left(\tau, \Delta, \mu_{0}, \mu\right) \cos (m \varphi)
$$

Here $\tau=z / l_{\text {tot }}$ is the dimensionless layer thickness, $z$ is the geometrical thickness of the layer, $l_{\text {tot }}=\left[n\left(\sigma_{\text {in }}+\sigma_{e l}\right)\right]^{-1}$ is the total mean free path, $n$ is the concentration of scatters, $\sigma_{e l}$ and $\sigma_{i n}$ are the elastic and inelastic scattering cross-sections, respectively, $\Delta$ is the energy loss, $\mu_{0}$ is the cosine of the incident zenith angle, $\mu$ is the cosine of the viewing zenith angle, $\varphi$ is the azimuthal angle between incident and sighting directions, and $\delta_{m m^{\prime}}$ is the Kronecker delta. Within the partial intensity approach, the functions $R^{m}$ can be expanded as follows:

$$
R^{m}\left(\tau, \Delta, \mu_{0}, \mu\right)=\sum_{k=0}^{\infty} R_{k}^{m}\left(\tau, \mu_{0}, \mu\right) x_{i n}^{k}(\Delta),
$$

where $x_{i n}^{0}(\Delta)=\delta(\Delta)$ is the Dirac function, $x_{i n}^{1}(\Delta)=x_{i n}(\Delta)$ is the NDIIMFP (DIIMFP normalized to unity area), and $x_{i n}^{k}(\Delta)$ is the spectrum of energy losses after $k$ successive inelastic scattering events. The latter is computed as the $k$-fold selfconvolution:

$$
x_{i n}^{k}(\Delta)=\int_{0}^{\Delta} x_{i n}^{k-1}(\varepsilon) x_{i n}(\Delta-\varepsilon) d \varepsilon
$$

and $\operatorname{DIIMFP}(\Delta)=x_{i n}(\Delta) / \operatorname{IMFP}$.

In practice, the summation in Eq. (2) is performed up to the $K$-th term, where $K$ is the maximum number of inelastic scattering collisions taken into account. The transmission function $T\left(\tau, \Delta, \mu_{0}, \mu, \varphi\right)$ and the photo-electron flux density $Q\left(\tau, \Delta, \mu_{0}, \mu, \varphi\right)$ are expanded analogously as in Eqs. (1) and (2) providing the partial intensities $T_{k}^{m}\left(\tau, \mu_{0}, \mu\right)$ and $Q_{k}^{m}\left(\tau, \mu_{0}, \mu\right)$. Further we omit the $m$-superscript for convenience. Note, that $R_{k}\left(\tau, \mu_{0}, \mu\right), T_{k}\left(\tau, \mu_{0}, \mu\right)$ and $Q_{k}\left(\tau, \mu_{0}, \mu\right)$ refer to the $k$-fold inelastically scattered particles, while $R_{0}\left(\tau, \mu_{0}, \mu\right), T_{0}\left(\tau, \mu_{0}, \mu\right)$ and $Q_{0}\left(\tau, \mu_{0}, \mu\right)$ refer to the elastically scattered electrons.

Assuming the Poisson stochastic process for multiple energy losses [15], the energy distribution of electrons passed path $\tau$ is written as

$$
L(\tau, \Delta)=\sum_{k=0}^{\infty} L_{k}(\tau, \Delta)=\sum_{k=0}^{\infty}\left\{\exp (-\tau) \frac{(1-\lambda)^{k} \tau^{k}}{k !} x_{i n}^{k}(\Delta)\right\}
$$

where $\lambda$ is the single scattering albedo, and $L_{k}(\tau, \Delta)$ is the distribution of energy losses after $k$-fold scattering as a function of $\tau[16,17]$.

To compute partial intensities we adopt the concept of invariant imbedding, which is due to Ambarzumian [18]. He derived an equation for reflection from a semi-infinite atmosphere by noting that the reflection function remains unchanged upon addition of a new layer. This technique was generalized by Chandrasekhar [19] for a finite layer. The extension of this method to the partial intensity approach is described in [20]. Derivation of equations for functions $R_{k}, T_{k}$, and $Q_{k}$ involves the following steps [21]:

1. add an infinitely thin layer to the sample;

2. consider single scattering processes in that layer which contribute to the change in $R_{k}, T_{k}$, and $Q_{k}$;

3. express $R_{k}, T_{k}$, and $Q_{k}$ functions for the system "sample + layer" through corresponding functions for the sample.

The resulting equations for elastically scattered electrons $(k=$ 0) read as follows:

$$
\begin{aligned}
& \frac{\partial}{\partial \tau} R_{0}\left(\tau, \mu_{0}, \mu\right)+\left(\frac{1}{\mu}+\frac{1}{\mu_{0}}\right) R_{0}\left(\tau, \mu_{0}, \mu\right) \\
& =\lambda x_{e l}^{-}\left(\mu_{0}, \mu\right)+\lambda \int_{0}^{1} x_{e l}^{+}\left(\mu_{0}, \mu^{\prime}\right) R_{0}\left(\tau, \mu^{\prime}, \mu\right) \frac{d \mu^{\prime}}{\mu^{\prime}} \\
& +\lambda \int_{0}^{1} R_{0}\left(\tau, \mu_{0}, \mu^{\prime}\right) x_{e l}^{+}\left(\mu^{\prime}, \mu\right) \frac{d \mu^{\prime}}{\mu^{\prime}} \\
& +\lambda \int_{0}^{1} \int_{0}^{1} R_{0}\left(\tau, \mu_{0}, \mu^{\prime}\right) x_{e l}^{-}\left(\mu^{\prime}, \mu^{\prime \prime}\right) R_{0}\left(\tau, \mu^{\prime \prime}, \mu\right) \frac{d \mu^{\prime}}{\mu^{\prime}} \frac{d \mu^{\prime \prime}}{\mu^{\prime \prime}},
\end{aligned}
$$

$$
\begin{aligned}
& \frac{\partial}{\partial \tau} T_{0}\left(\tau, \mu_{0}, \mu\right)+\frac{1}{\mu} T_{0}\left(\tau, \mu_{0}, \mu\right) \\
& =\lambda L_{0}\left(\frac{\tau}{\mu_{0}}\right) \cdot x_{e l}^{+}\left(\mu_{0}, \mu\right)+\lambda \int_{0}^{1} T_{0}\left(\tau, \mu_{0}, \mu^{\prime}\right) x_{e l}^{+}\left(\mu^{\prime}, \mu\right) \frac{d \mu^{\prime}}{\mu^{\prime}} \\
& +\lambda L_{0}\left(\frac{\tau}{\mu_{0}}\right) \cdot \int_{0}^{1} x_{e l}^{-}\left(\mu_{0}, \mu^{\prime}\right) R_{0}\left(\tau, \mu^{\prime}, \mu\right) \frac{d \mu^{\prime}}{\mu^{\prime}} \\
& +\lambda \int_{0}^{1} \int_{0}^{1} T_{0}\left(\tau, \mu_{0}, \mu^{\prime}\right) x_{e l}^{-}\left(\mu^{\prime}, \mu^{\prime \prime}\right) R_{0}\left(\tau, \mu^{\prime \prime}, \mu\right) \frac{d \mu^{\prime}}{\mu^{\prime}} \frac{d \mu^{\prime \prime}}{\mu^{\prime \prime}},
\end{aligned}
$$




$$
\begin{aligned}
& \frac{\partial}{\partial \tau} Q_{0}\left(\tau, \mu_{0}, \mu\right)+\frac{1}{\mu} Q_{0}\left(\tau, \mu_{0}, \mu\right) \\
& =\lambda_{\gamma} F^{-}\left(\mu_{0}, \mu\right)+\lambda \int_{0}^{1} Q_{0}\left(\tau, \mu_{0}, \mu^{\prime}\right) x_{e l}^{+}\left(\mu^{\prime}, \mu\right) \frac{d \mu^{\prime}}{\mu^{\prime}} \\
& +\lambda_{\gamma} \int_{0}^{1} F^{+}\left(\mu_{0}, \mu^{\prime}\right) R_{0}\left(\tau, \mu^{\prime}, \mu\right) \frac{d \mu^{\prime}}{\mu^{\prime}} \\
& +\lambda \int_{0}^{1} \int_{0}^{1} Q_{0}\left(\tau, \mu_{0}, \mu^{\prime}\right) x_{e l}^{-}\left(\mu^{\prime}, \mu^{\prime \prime}\right) R_{0}\left(\tau, \mu^{\prime \prime}, \mu\right) \frac{d \mu^{\prime}}{\mu^{\prime}} \frac{d \mu^{\prime \prime}}{\mu^{\prime \prime}} .
\end{aligned}
$$

Here $x_{e l}\left(\mu_{0}, \mu\right)$ is the azimuthal expansion coefficient of the differential elastic scattering cross-section normalized to unity area while $F$ is the azimuthal expansion coefficient of the differential photo-ionization cross section, $x_{e l}^{+}, x_{e l}^{-}$and $F^{+}, F^{-}$are defined as

$$
\begin{cases}x_{e l}^{+}\left(\mu_{0}, \mu\right)=x_{e l}\left(\mu_{0}, \mu\right), & \operatorname{sign}\left(\mu_{0} \cdot \mu\right)=1, \\ x_{e l}^{-}\left(\mu_{0}, \mu\right)=x_{e l}\left( \pm \mu_{0}, \mp \mu\right), & \operatorname{sign}\left(\mu_{0} \cdot \mu\right)=-1\end{cases}
$$

The equations for $k$-fold inelastically scattered electrons are given by

$$
\begin{aligned}
& \frac{\partial}{\partial \tau} R_{k}\left(\tau, \mu_{0}, \mu\right)+\left(\frac{1}{\mu}+\frac{1}{\mu_{0}}\right) R_{k}\left(\tau, \mu_{0}, \mu\right) \\
& =(1-\lambda)\left(\frac{1}{\mu_{0}}+\frac{1}{\mu}\right) R_{k-1}\left(\tau, \mu_{0}, \mu\right) \\
& +\lambda \int_{0}^{1} x_{e l}^{+}\left(\mu_{0}, \mu^{\prime}\right) R_{k}\left(\tau, \mu^{\prime}, \mu\right) \frac{d \mu^{\prime}}{\mu^{\prime}} \\
& +\lambda \int_{0}^{1} R_{k}\left(\tau, \mu_{0}, \mu^{\prime}\right) x_{e l}^{+}\left(\mu^{\prime}, \mu\right) \frac{d \mu^{\prime}}{\mu^{\prime}} \\
& +\lambda \int_{0}^{1} \int_{0}^{1} R_{k}\left(\tau, \mu_{0}, \mu^{\prime}\right) x_{e l}^{-}\left(\mu^{\prime}, \mu^{\prime \prime}\right) R_{0}\left(\tau, \mu^{\prime \prime}, \mu\right) \frac{d \mu^{\prime \prime}}{\mu^{\prime \prime}} \frac{d \mu^{\prime}}{\mu^{\prime}} \\
& +\lambda \int_{0}^{1} \int_{0}^{1} R_{0}\left(\tau, \mu_{0}, \mu^{\prime}\right) x_{e l}^{-}\left(\mu^{\prime}, \mu^{\prime \prime}\right) R_{k}\left(\tau, \mu^{\prime \prime}, \mu\right) \frac{d \mu^{\prime \prime}}{\mu^{\prime \prime}} \frac{d \mu^{\prime}}{\mu^{\prime}} \\
& +\lambda \sum_{i=1}^{k-1}\left\{\int_{0}^{1} \int_{0}^{1} R_{i}\left(\tau, \mu_{0}, \mu^{\prime}\right) x_{e l}^{-}\left(\mu^{\prime}, \mu^{\prime \prime}\right) R_{k-i}\left(\tau, \mu^{\prime \prime}, \mu\right) \frac{d \mu^{\prime}}{\mu^{\prime}} \frac{d \mu^{\prime \prime}}{\mu^{\prime \prime}}\right\},
\end{aligned}
$$

$$
\begin{aligned}
& \frac{\partial}{\partial \tau} T_{k}\left(\tau, \mu_{0}, \mu\right)+\frac{1}{\mu} T_{k}\left(\tau, \mu_{0}, \mu\right) \\
& =\frac{1-\lambda}{\mu} T_{k-1}\left(\tau, \mu_{0}, \mu\right)+\lambda L_{k}\left(\frac{\tau}{\mu_{0}}\right) \cdot x_{e l}^{+}\left(\mu_{0}, \mu\right) \\
& +\lambda \int_{0}^{1} T_{k}\left(\tau, \mu_{0}, \mu^{\prime}\right) x_{e l}^{+}\left(\mu^{\prime}, \mu\right) \frac{d \mu^{\prime}}{\mu^{\prime}} \\
& +\lambda \sum_{i=0}^{k}\left\{L_{i}\left(\frac{\tau}{\mu_{0}}\right) \cdot \int_{0}^{1} x_{e l}^{-}\left(\mu_{0}, \mu^{\prime}\right) R_{k-i}\left(\tau, \mu^{\prime}, \mu\right) \frac{d \mu^{\prime}}{\mu^{\prime}}\right\} \\
& +\lambda \int_{0}^{1} \int_{0}^{1} T_{k}\left(\tau, \mu_{0}, \mu^{\prime}\right) x_{e l}^{-}\left(\mu^{\prime}, \mu^{\prime \prime}\right) R_{0}\left(\tau, \mu^{\prime \prime}, \mu\right) \frac{d \mu^{\prime}}{\mu^{\prime}} \frac{d \mu^{\prime \prime}}{\mu^{\prime \prime}} \\
& +\lambda \sum_{i=0}^{k-1}\left\{\int_{0}^{1} \int_{0}^{1} T_{i}\left(\tau, \mu_{0}, \mu^{\prime}\right) x_{e l}^{-}\left(\mu^{\prime}, \mu^{\prime \prime}\right) R_{k-i}\left(\tau, \mu^{\prime \prime}, \mu\right) \frac{d \mu^{\prime}}{\mu^{\prime}} \frac{d \mu^{\prime \prime}}{\mu^{\prime \prime}}\right\},
\end{aligned}
$$

$$
\begin{aligned}
& \frac{\partial}{\partial \tau} Q_{k}\left(\tau, \mu_{0}, \mu\right)+\frac{1}{\mu} Q_{k}\left(\tau, \mu_{0}, \mu\right) \\
& =\frac{1-\lambda}{\mu} Q_{k-1}\left(\tau, \mu_{0}, \mu\right)+\lambda_{\gamma} \int_{0}^{1} F^{+}\left(\mu_{0}, \mu^{\prime}\right) R_{k}\left(\tau, \mu^{\prime}, \mu\right) \frac{d \mu^{\prime}}{\mu^{\prime}} \\
& +\lambda \int_{0}^{1} Q_{k}\left(\tau, \mu_{0}, \mu^{\prime}\right) x_{e l}^{+}\left(\mu^{\prime}, \mu\right) \frac{d \mu^{\prime}}{\mu^{\prime}} \\
& +\lambda \int_{0}^{1} \int_{0}^{1} Q_{k}\left(\tau, \mu_{0}, \mu^{\prime}\right) x_{e l}^{-}\left(\mu^{\prime}, \mu^{\prime \prime}\right) R_{0}\left(\tau, \mu^{\prime \prime}, \mu\right) \frac{d \mu^{\prime}}{\mu^{\prime}} \frac{d \mu^{\prime \prime}}{\mu^{\prime \prime}} \\
& +\lambda \sum_{i=0}^{k-1}\left\{\int_{0}^{1} \int_{0}^{1} Q_{i}\left(\tau, \mu_{0}, \mu^{\prime}\right) x_{e l}^{-}\left(\mu^{\prime}, \mu^{\prime \prime}\right) R_{k-i}\left(\tau, \mu^{\prime \prime}, \mu\right) \frac{d \mu^{\prime}}{\mu^{\prime}} \frac{d \mu^{\prime \prime}}{\mu^{\prime \prime}}\right\} .
\end{aligned}
$$

Eqs. (4)-(10) are discretized in the angular domain by defining a set of Gaussian quadrature points and weights in the zenith direction. We are led to a differential matrix equations which can be solved by using either the backward differentiation formula (BDF) [22] or the matrix exponential formalism [23]. In [20] both techniques are validated against Monte-Carlo simulations and experimental angular distributions of scattered electrons. An agreement within $1 \%$ is obtained between them, while the computational time for solving Eqs. (4)-(10) is less than a second on Intel Xeon CPU E5-1620 3.60GHz (the performance can be further enhanced by using acceleration techniques for the discrete ordinate method [24] or by parallel computing [25]). Hence, the proposed technique for partial intensities computations is robust and can be effectively used in the direct search methods of nonlinear optimization.

\section{REELS and PES spectra computation for multi-layer sys- tems}

To take into account surface excitations, we consider a twolayer system containing a surface layer (designated by the subscript "S") and a semi-infinite bulk layer (designated by the subscript "B"), as shown in Fig. 1a. The reflection function $R_{B S}$ for two-layer systems can be expressed as follows:

$$
\begin{aligned}
& R_{B S}\left(\tau_{S}, \Delta, \mu_{0}, \mu, \varphi\right)=R_{S}\left(\tau_{S}, \Delta, \mu_{0}, \mu, \varphi\right) \\
& +\int_{0}^{\Delta} d \varepsilon \int_{0}^{\varepsilon} d \varepsilon^{\prime} \int_{0}^{2 \pi} \int_{0}^{1} \int_{0}^{2 \pi} \int_{0}^{1} T_{S}\left(\tau_{S}, \Delta-\varepsilon, \mu_{0}, \mu^{\prime}, \varphi^{\prime}\right) \\
& \cdot R_{B}\left(\varepsilon-\varepsilon^{\prime}, \mu^{\prime}, \mu^{\prime \prime}, \varphi^{\prime \prime}-\varphi^{\prime}\right) \\
& \cdot T_{S}\left(\tau_{S}, \varepsilon^{\prime}, \mu^{\prime \prime}, \mu, \varphi-\varphi^{\prime \prime}\right) \frac{d \mu^{\prime}}{\mu^{\prime}} d \varphi^{\prime} \frac{d \mu^{\prime \prime}}{\mu^{\prime \prime}} d \varphi^{\prime \prime},
\end{aligned}
$$

where $R_{S}$ and $T_{S}$ are the reflection function and transmission function for the surface layer, respectively, while $R_{B}$ stands for the reflection function of the semi-infinite bulk layer $\left(\tau_{B} \rightarrow \infty\right)$. Analogously, the photo-electron flux density $Q_{B S}$ for the two- 
layer system (see Fig. 1c) reads as follows:

$$
\begin{aligned}
& Q_{B S}\left(\tau_{S}, \Delta, \mu_{0}, \mu, \varphi\right)=Q_{S}\left(\tau_{S}, \Delta, \mu_{0}, \mu, \varphi\right) \\
& +\int_{0}^{\Delta} d \varepsilon \int_{0}^{2 \pi} \int_{0}^{1} Q_{B}\left(\Delta-\varepsilon, \mu_{0}, \mu^{\prime}, \varphi^{\prime}\right) \\
& \cdot T_{S}\left(\tau_{S}, \varepsilon, \mu^{\prime}, \mu, \varphi-\varphi^{\prime}\right) \frac{d \mu^{\prime}}{\mu^{\prime}} d \varphi^{\prime} \\
& +\int_{0}^{\Delta} d \varepsilon \int_{0}^{\varepsilon} d \varepsilon^{\prime} \int_{0}^{2 \pi} \int_{0}^{1} \int_{0}^{2 \pi} \int_{0}^{1} Q_{S}\left(\tau_{S}, \Delta-\varepsilon, \mu_{0}, \mu^{\prime}, \varphi^{\prime}\right) \\
& \cdot R_{B}\left(\varepsilon-\varepsilon^{\prime}, \mu^{\prime}, \mu^{\prime \prime}, \varphi^{\prime \prime}-\varphi^{\prime}\right) \\
& \cdot T_{S}\left(\tau_{S}, \varepsilon^{\prime}, \mu^{\prime \prime}, \mu, \varphi-\varphi^{\prime \prime}\right) \frac{d \mu^{\prime}}{\mu^{\prime}} d \varphi^{\prime} \frac{d \mu^{\prime \prime}}{\mu^{\prime \prime}} d \varphi^{\prime \prime} .
\end{aligned}
$$

Here $Q_{S}$ and $Q_{B}$ are the photo-electron flux densities for the surface and bulk layers, respectively.

In the energy range $1-50 \mathrm{keV}$ of the probing beam, the thickness of the surface layer is much less than the transport mean free path. Therefore, the trajectories in the surface layer can be approximated by straight lines. As shown in [26], the error induced by this assumption does not exceed $3 \%$ excluding glancing sighting angles. Consequently, Eqs. (11) and (12) can be simplified as follows:

$$
\begin{aligned}
& R_{B S}\left(\tau_{S}, \Delta, \mu_{0}, \mu, \varphi\right)=R_{S}\left(\tau_{S}, \Delta, \mu_{0}, \mu, \varphi\right) \\
& +\int_{0}^{\Delta} R_{B}\left(\Delta-\varepsilon, \mu_{0}, \mu, \varphi\right) L\left(\tau_{S}\left(\frac{1}{\mu}+\frac{1}{\mu_{0}}\right), \varepsilon\right) d \varepsilon \\
& \quad Q_{B S}\left(\tau_{S}, \Delta, \mu_{0}, \mu, \varphi\right)=Q_{S}\left(\tau_{S}, \Delta, \mu_{0}, \mu, \varphi\right) \\
& \quad+\int_{0}^{\Delta} Q_{B}\left(\Delta-\varepsilon, \mu_{0}, \mu, \varphi\right) L\left(\frac{\tau_{S}}{\mu}, \varepsilon\right) d \varepsilon .
\end{aligned}
$$

In some cases it is beneficial to increase the number of fitting parameters in order to reduce the residual between computed and measured spectra. In particular, an additional "intermediate" layer (designated by the subscript "G") between surface and bulk can be assumed. The reflection function for the three-layer system (see Fig. 1b) can be derived in the same manner as that for the two layer system and is given by

$$
\begin{aligned}
& R_{B G S}\left(\tau_{G}+\tau_{S}, \Delta, \mu_{0}, \mu, \varphi\right)= \\
& =R_{G S}\left(\tau_{G}+\tau_{S}, \Delta, \mu_{0}, \mu, \varphi\right) \\
& +\int_{0}^{\Delta} \int_{0}^{\varepsilon^{\prime}} R_{B}\left(\Delta-\varepsilon^{\prime}, \mu_{0}, \mu, \varphi\right) \\
& \cdot L\left[\tau_{S}\left(\frac{1}{\mu}+\frac{1}{\mu_{0}}\right), \varepsilon^{\prime}-\varepsilon^{\prime \prime}\right] \cdot L\left[\tau_{G}\left(\frac{1}{\mu}+\frac{1}{\mu_{0}}\right), \varepsilon^{\prime \prime}\right] d \varepsilon^{\prime \prime} d \varepsilon^{\prime} .
\end{aligned}
$$

\section{Retrieval of inelastic scattering properties}

\subsection{Algorithm for NDIIMFP and NDSEP extraction}

Unknown NDIIMFP/NDSEP functions are sought in the following form:

$$
x_{\text {in }}(\Delta)=\sum_{i=1}^{N_{p l}} \lambda_{p l i} x_{p l i}(\Delta)+\sum_{j=1}^{N_{\text {ion }}} \lambda_{\text {ion } j} x_{\text {ion } j}(\Delta),
$$

where $N_{p l}$ and $N_{i o n}$ are the numbers of plasmons and ionization processes taken into account, respectively, $\lambda_{p l i}$ and $\lambda_{\text {ion } j}$ are the corresponding weights, while $x_{p l}$ and $x_{i o n}$ are basic functions for plasmons and ionization processes, respectively. The expression for $x_{p l}$ is given by

$$
x_{p l i}(\Delta)=\frac{A_{p l i} \Delta^{\beta}}{\left(\Delta^{2}-\varepsilon_{p l i}^{2}\right)^{2}+b_{i}^{4-\alpha} \Delta^{\alpha}} .
$$

This formula is a modification of the dispersion relation in solids and can be derived within linear response theory [2]. The coefficient $A_{p l}$ is required for normalization of $x_{p l}$ to unity area, $\varepsilon_{p l}$ is the effective plasmon energy corresponded to the plasmon peak position, $b$ is the attenuation coefficient which controls the width of the function, while $\alpha$ and $\beta$ are tuning parameters related to the asymmetry of $x_{p l}$. For $x_{i o n}$ we use the modified Rutherford formula:

$$
x_{\text {ion } j}(\Delta)=\frac{A_{\text {ion } j}}{\Delta^{2+a}} \eta\left(\Delta-J_{\text {ion } j}\right) .
$$

Here $\eta$ is the Heaviside step function, $J_{\text {ion } j}$ is the $j$-th ionization potential, while $a$ is the coefficient accounting for the electron screening of a Coulomb potential.

The values for $J_{i o n} j$ are taken from [27]. The elastic scattering parameters are taken from NIST Elastic-Scattering CrossSection Database - Version 3.2 [28] based on the code ELSEPA [29]. The IMFP values are taken from NIST Electron InelasticMean-Free-Path Database - Version 1.2 [1], which encapsulates the experimental optical data as well as IMFP values computed with the predictive TPP-2M formula [30].

To account for the instrumental energy broadening, all calculated spectra are convolved with a slit function of the energyanalyzer. The convolution procedure transforms zero-width elastic peaks $(k=0)$ of Eq. (2) to the Gaussian-like form, smoothes the whole spectrum and leads to a better agreement between computed spectra and experimental data. For PES we also take into account effects described in [31]. The rest parameters including the surface layer thickness are obtained by using either the fitting procedure or global optimization methods which minimize the residual between computed and measured spectra. Note, that local optimization techniques may fail here since the residual has many local minima. Computations of REELS and PES spectra are performed using the solution technique described in Section 2.

There is a controversy regarding the photo-electron energy losses due to so-called intrinsic plasmon excitations. In [32, 33 ] the intrinsic plasmon excitations related to electron photoionization effects are considered. In this paper, computations of PES spectra are performed without accounting for intrinsic plasmon excitations since plasmons are essentially collective processes while ionization is a local process in our model.

\subsection{DIIMFP and DSEP extraction from $M g$, Al and Si REELS spectra}

The procedure outlined above was implemented and applied to experimental $\mathrm{Mg}, \mathrm{Al}$ and $\mathrm{Si}$ REELS spectra taken from $[7,34,35]$. The two-layer model is used to take into account surface excitations. The reflection function $R_{B}$ is calculated involving Eqs. (1), (2), (4) and (8). Figures 2, 3 and 4 illustrate 

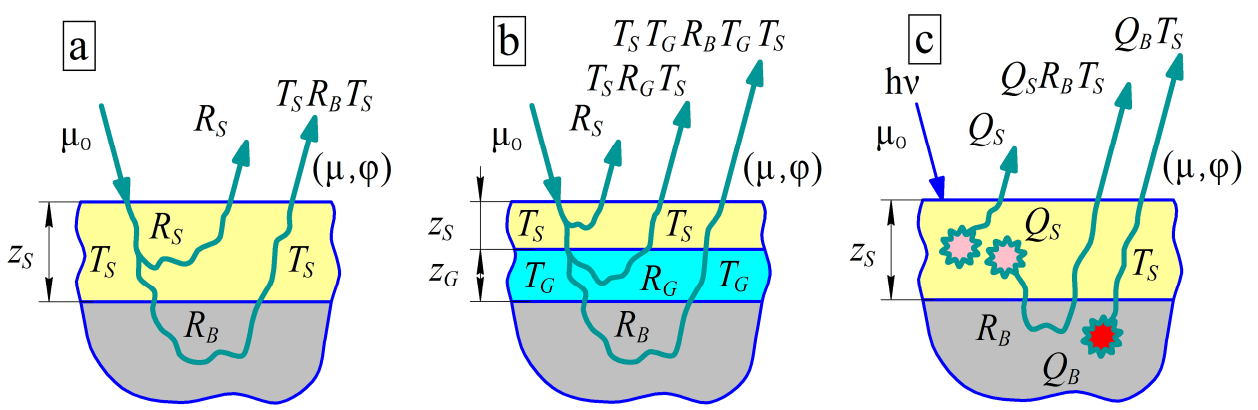

Figure 1: Multilayer models of a sample for REELS: (a) two-layer model, (b) three-layer model; and for PES: (c) two-layer model. The arrows represent processes defined by reflection $(\mathrm{R})$, transmission $(\mathrm{T})$ and photo-electron flux density $(\mathrm{Q})$ functions forming a signal from a multilayer system.

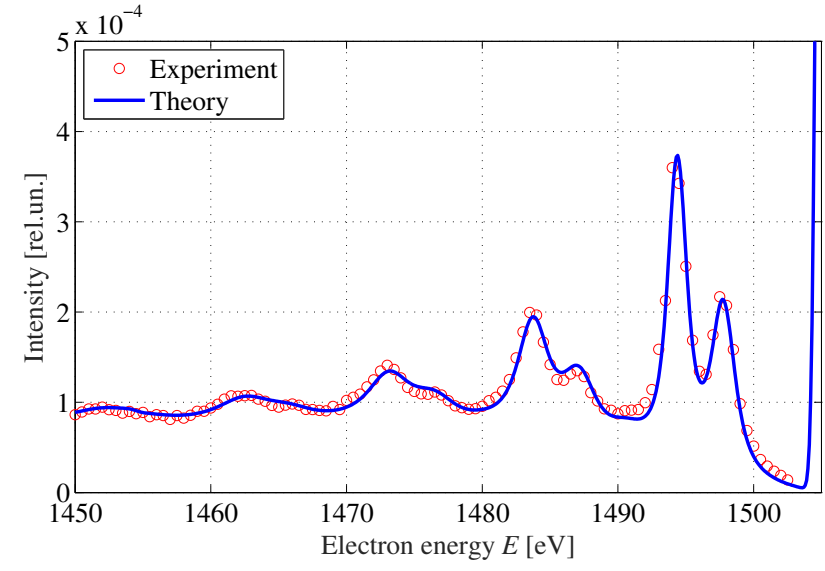

Figure 2: Comparison of experimental REELS spectra from [34] of Mg with calculated spectra. The initial energy is $1505 \mathrm{eV}$. The average relative error is $5.4 \%$.

the result of fitting. For all considered cases the maximum discrepancy is of about $5 \%$ while the average discrepancy is of about $1 \%$ the range of energy losses $0-100 \mathrm{eV}$. The retrieved normalized DIIMFPs and DSEPs for $\mathrm{Mg}, \mathrm{Al}$ and $\mathrm{Si}$ are shown in Fig. 5, 6 and 7, respectively. The surface plasmon energy is lower than the bulk plasmon energy approximately by factor $\sqrt{2}$ [36]. Note, that it is sufficient to take into account two inelastic collisions in the surface layer to get a good agreement with measured spectra.

\subsection{Obtaining DIIMFP and DSEP functions for $N b$ from REELS spectra}

Here we address the problem of DIIMFP retrieval for $\mathrm{Nb}$. For analysis we take five REELS spectra of $\mathrm{Nb}$ measured at several probing energies $E_{0}: 5 \mathrm{keV}, 10 \mathrm{keV}, 20 \mathrm{keV}, 25 \mathrm{keV}$ and $40 \mathrm{keV}$ (the measurements have been performed by M.Went and M.Vos (Australian National University) [39]). Retrieval of DIIMFP and DSEP is performed within a three-layer model [40]. The reflection function is simulated using Eq. (15). Extracted functions together with the normilized DIIMFP taken from [37] are shown in Fig. 9 while the measured and computed spectra for some probing energies are presented in Fig. 8.
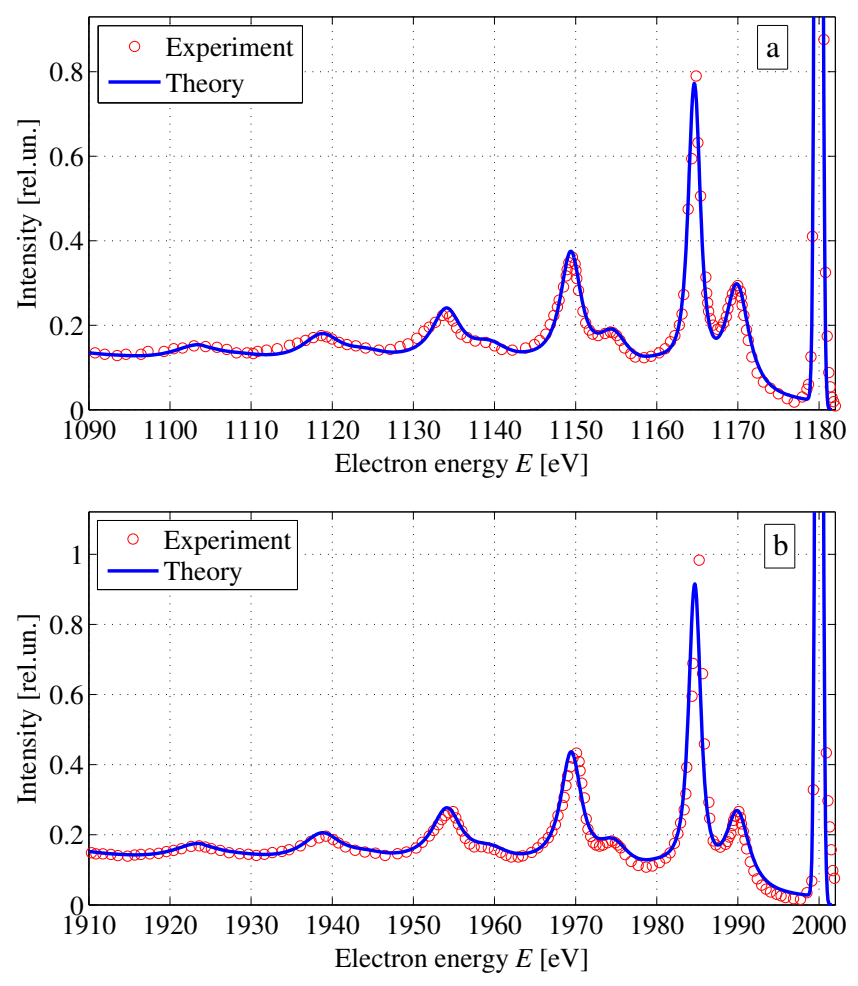

Figure 3: Comparison of experimental REELS spectra from [7] of Al with calculated spectra. The primary electron energies are $1180 \mathrm{eV}$ (a) and $2000 \mathrm{eV}$ (b); the average relative errors are $6.0 \%$ (a) and $8.2 \%$ (b), respectively.

The relative difference in the REELS spectra does not exceed $5 \%$. Figure 10 shows the thicknesses of surface and intermediate layers ( $\tau_{S}$ and $\tau_{G}$, respectively) as functions of $E_{0}$. The width of the surface scattering zone reduces with $E_{0}$ approximately as $E_{0}^{-0.5}$. This is consistent with the theory given in [41].

The necessity of using the three-layer model deserves some words of explanation. In our previous studies, we saw that the interpretation of $\mathrm{Nb}$ REELS spectra can be performed within a two-layer model. In this case, the residual is small only if the basic functions (17), (18) depend on $E_{0}$. This somewhat com- 


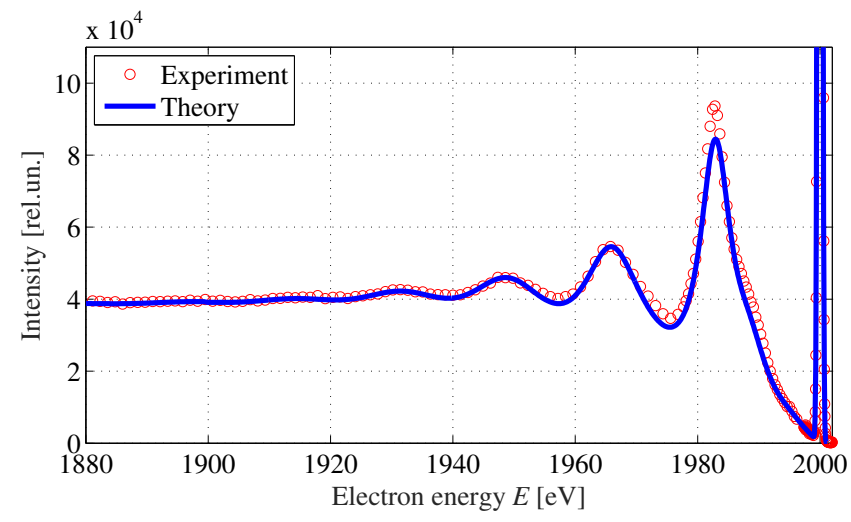

Figure 4: Comparison of experimental REELS spectra of Si with calculated spectra. The primary electron energy is $2000 \mathrm{eV}$. The average relative error is $3.5 \%$.

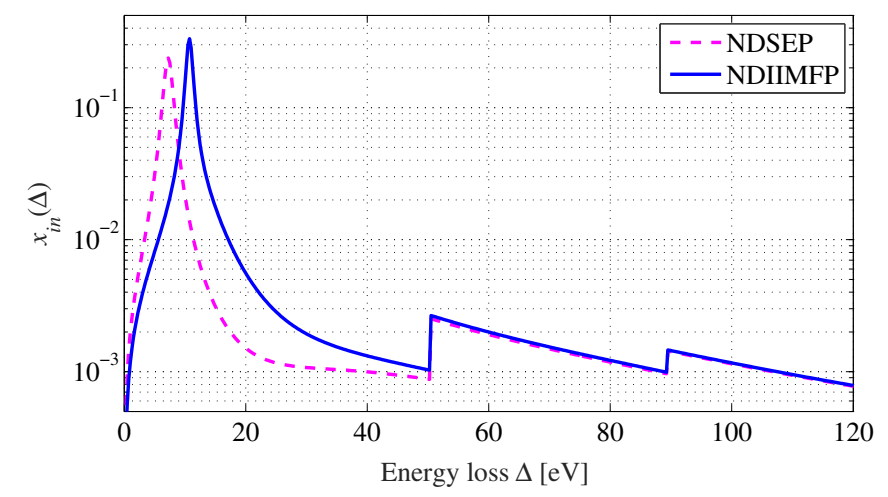

Figure 5: Retrieved NDIIMFP and NDSEP of an outgoing $1505 \mathrm{eV}$ electron for $\mathrm{Mg}$ sample.

plicates the retrieval since the space of DIIMFP functions becomes significantly larger and it is more difficult to find a global minimum of the residual. The DIIMFP obtained within threelayer models can be regarded as an intermediate result. In particular, it can be transformed to that corresponded to two-layer models by introducing an effective $\mathrm{DSEP}_{e}$ which is a weighted sum of DSEP in surface and intermediate layers:

$$
\operatorname{DSEP}_{e}(\Delta)=\frac{\tau_{G} \operatorname{DSEP}_{G}(\Delta)+\tau_{S} \operatorname{DSEP}_{S}(\Delta)}{\tau_{G}+\tau_{S}},
$$

In addition, REELS spectra of relatively low resolution can be interpreted within a one layer model, as it was shown in [42]. Thus, an optimal number of layers depend on the initial problem and the retrieval strategy.

\subsection{Retrieval of DIIMFP and DSEP for Be and $W$ from PES spectra}

Bearing in mind, that the number of PES spectra available for analysis exceeds that of REELS by order of magnitude [4345], it is beneficial to have an algorithm for retrieving DIIMFP and DSEP functions from PES spectra. At the same time, it

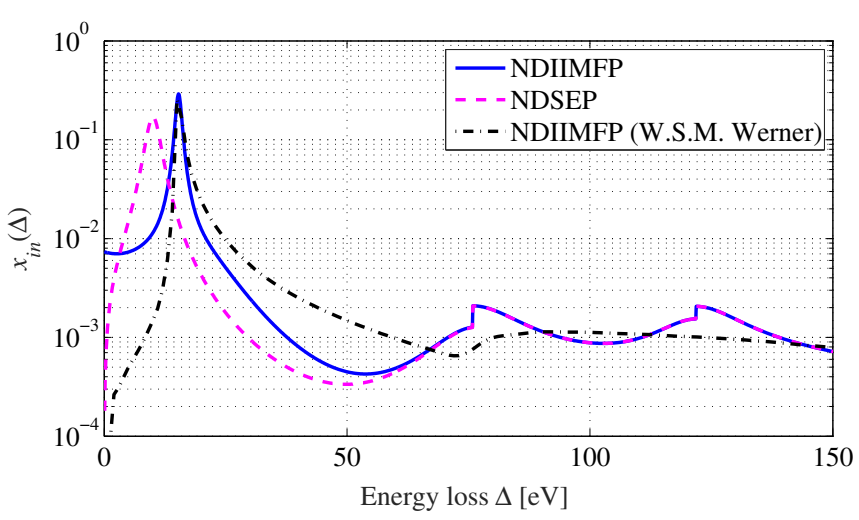

Figure 6: Comparison of retrieved NDIIMFP and NDSEP of an outgoing $2000 \mathrm{eV}$ electron for Al sample with NDIIMFP from [37].

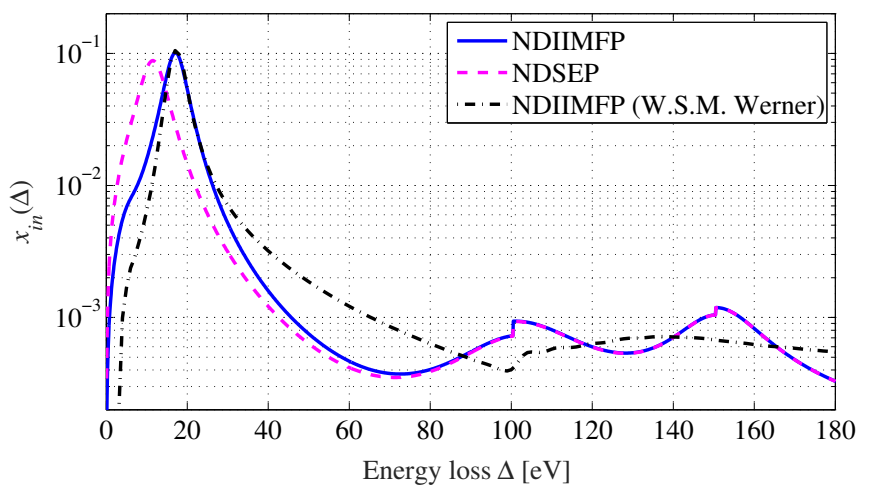

Figure 7: Comparison of retrieved NDIIMFP and NDSEP for Si sample with NDIIMFP from [38]. The primary electron energy is $2000 \mathrm{eV}$.

is quite challenging to develop such an algorithm based on the direct numerical extraction schemes since the impacts of several molecular electronic transition are overlapped in the PES spectrum.

In this section we extract DIIMFP and DSEP information from PES spectra by means of the fitting procedure. Our retrieval algorithm for PES is similar to that for REELS. The computations of PES spectra are performed in three steps:

1. we calculate $Q_{k}\left(\tau, \mu_{0}, \mu\right)$ function for each considered molecular electronic transition by solving a system of Eqs. (4)(10);

2. given $Q_{k}\left(\tau, \mu_{0}, \mu\right)$, we compute an energy loss spectrum for each molecular electronic transition using expansions analogous to Eqs. (1) and (2), and

3. we sum up impacts from all transitions (levels).

For Beryllium we consider $Q_{k}\left(\tau, \mu_{0}, \mu\right)$ functions related to the photo-electron emission from the energy level $1 s_{1 / 2}$. At least 7 inelastic scattering events must be taken into account to correctly reproduce the PES spectrum (see Fig. 11). For Tungsten we consider a set of $Q_{k}\left(\tau, \mu_{0}, \mu\right)$ functions related to the 

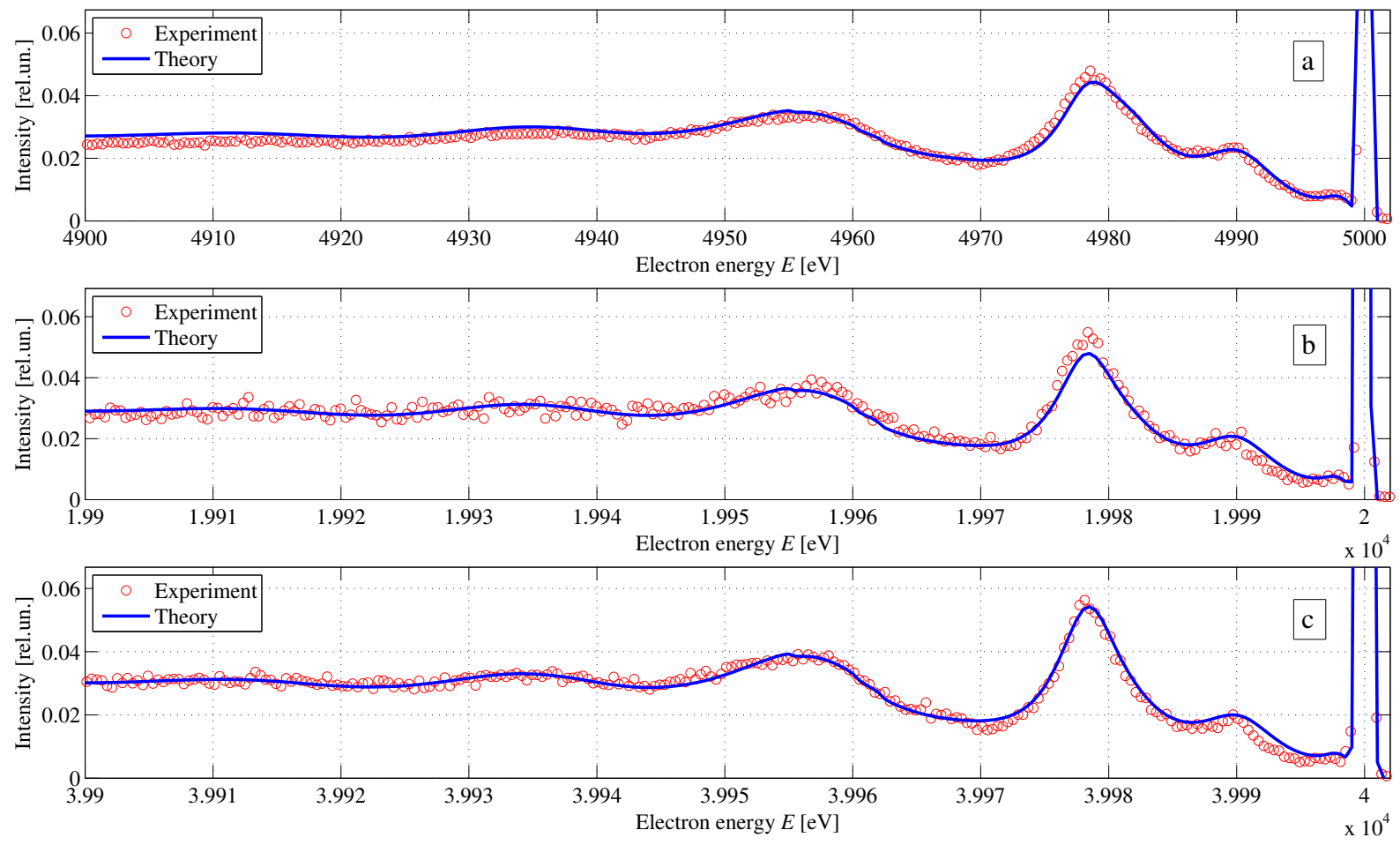

Figure 8: Comparison of experimental REELS spectra of $\mathrm{Nb}[39,40]$ with calculated spectra. The primary electron energies are $5 \mathrm{keV}$ (a), $20 \mathrm{keV}$ (b) and $40 \mathrm{keV}$ (c); the average relative errors are $5.6 \%$ (a), $7.3 \%$ (b) and $6.8 \%$ (c), respectively.

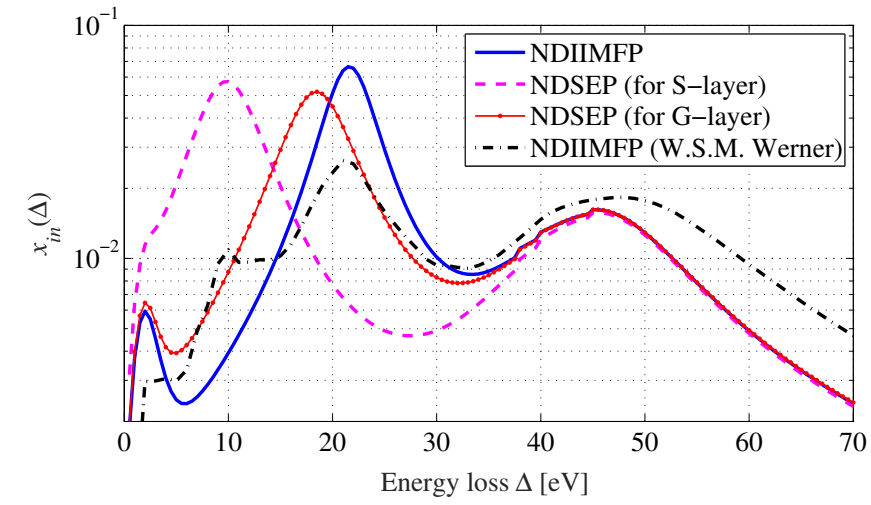

Figure 9: Comparison of retrieved NDSEP for surface (S) and intermediate $(\mathrm{G})$ layers and NDIIMFP of an outgoing $5 \mathrm{keV}$ electron for $\mathrm{Nb}$ sample for with NDIIMFP from [37].

photo-electron emission from levels $5 s_{1 / 2}, 5 p_{1 / 2}, 5 p_{3 / 2}, 4 f_{5 / 2}$, $4 f_{7 / 2}$. For adequate description of emission from these levels, 7 inelastic scattering collisions must be taken into account.

Figures 12 and 14 show the extracted DIIMFPs for Be and $\mathrm{W}$, respectively, obtained from PES spectra. In addition, we plot the DIIMFP taken from [37] . The extraction is performed within the two-layer model.

Computed PES spectra and experimental data are shown in Fig. 11 and Fig. 13 for Be and W, respectively. The agreement between experimental data and computed spectra is within 5\%

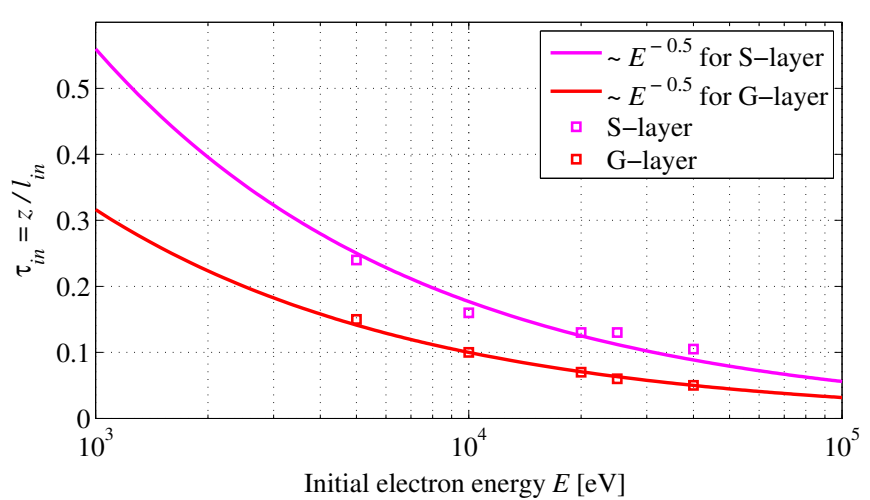

Figure 10: $\mathrm{Nb}$ surface excitation parameters $\left(\tau_{S}, \tau_{G}\right)$ as functions of the electron energy.

over the entire energy loss range of interest.

Note that each peak in the extracted DIIMFP is related to the specific physical process (whether it is a plasmon excitation or ionization of $5 s_{1 / 2}, 5 p_{1 / 2}$ and $5 p_{3 / 2}$ shells). The DIIMFP extracted by the direct numerical deconvolution (e.g., see [37] ) has five peaks in the energy loss range $0-50 \mathrm{eV}$ with insufficiently clear physical meaning. However, both DIIMFPs reproduce energy loss spectra. This is due to the fact that retrieval of DIIMFPs from REELS is a severely ill-posed problem and there is no unique solution to it. In this regard, a regularization technique employing physical models is highly required. 


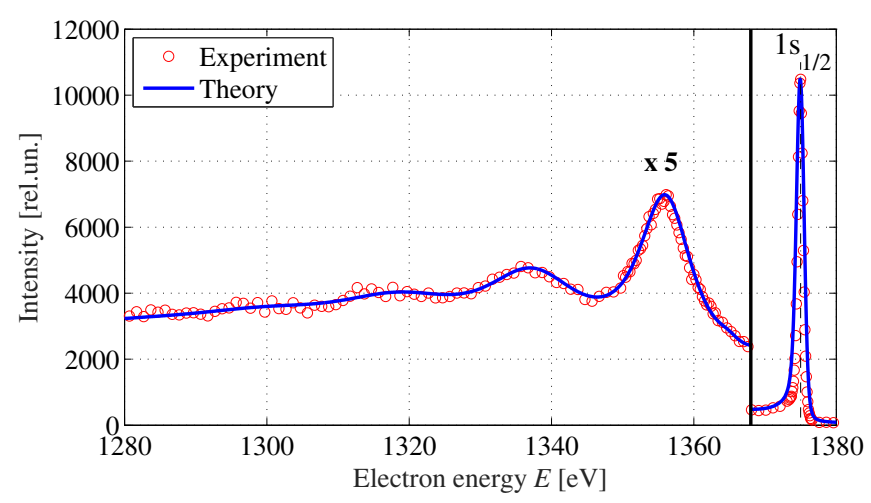

Figure 11: Comparison of experimental PES spectra [45] of Be with calculated spectra. The average relative error is $2.4 \%$.

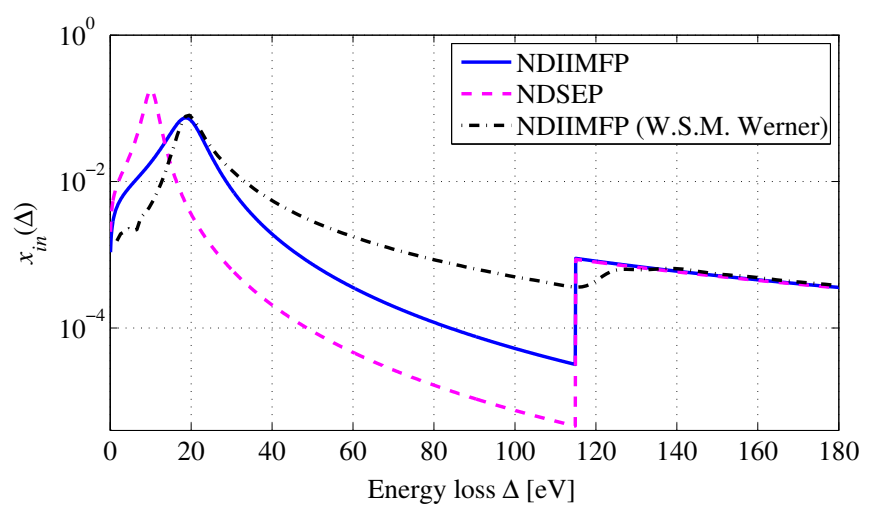

Figure 12: Comparison of retrieved NDIIMFP and NDSEP for Be sample for PES spectra with NDIIMFP from [37] .

\subsection{PES for Si, Al, Mg and Nb, computed with DIIMFPs ob- tained from REELS spectra}

To validate the DIIMFP and DSEP functions obtained from REELS, we use them to compute PES spectra. For $\mathrm{Si}, \mathrm{Al}$ and $\mathrm{Mg}$ one has to sum up $2 p_{1 / 2}, 2 p_{3 / 2}$ and $2 s_{1 / 2}$ lines. For $\mathrm{Nb}$ one has to sum up $3 d_{3 / 2}, 3 d_{5 / 2}, 3 p_{1 / 2}, 3 p_{3 / 2}$ and $3 s_{1 / 2}$ lines. Figures 15, 16, 17 and 18 illustrate experimental PES spectra for $\mathrm{Mg}, \mathrm{Al}, \mathrm{Si}$ and $\mathrm{Nb}$, respectively, as well as spectra, computed using DIIMFPs obtained from REELS. Good agreement is obtained, what proves the consistency of the proposed method.

\section{Summary}

We presented a unified approach for computing REELS and PES spectra. It is based on the invariant imbedding equations solved by using the discrete ordinate method and the backward differential formula. This approach provides a numerical solution of transport equations without $a d$ hoc assumptions and simplifications. Note that the forward model is robust, e.g. the computational time for one PES spectrum simulation does not exceed $0.1 \mathrm{sec}$ on Intel Xeon CPU E5-1620 3.60GHz.

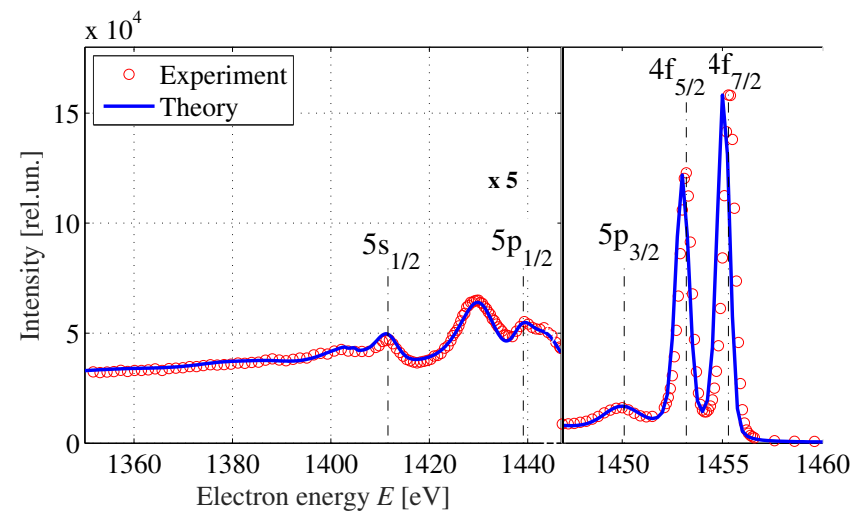

Figure 13: Comparison of experimental PES spectra [45] of W with calculated spectra. The average relative error is $3.3 \%$.

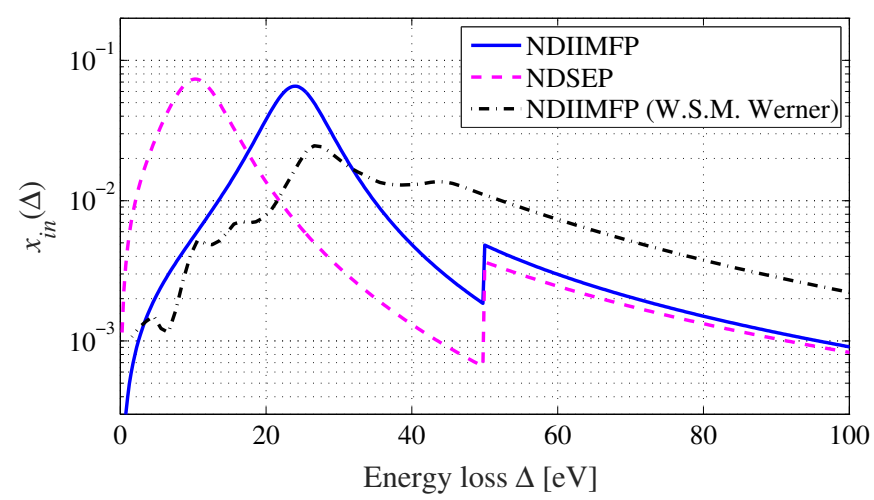

Figure 14: The same as Fig. 12 except for W.

Using this approach as a tool to account for multiple scattering effects, we have developed the algorithm for DIIMFP and DSEP extraction from REELS and PES spectra. The desired functions are parametrized on the base of linear respond theory. Unknown parameters are found by means of the fitting procedure, which minimizes the residual between simulated spectra and measurements. Unlike direct numerical inversion schemes, the proposed method is stable and provides physically relevant results.

To account for surface plasmons, the multi-layer model is used. In particular, the DIIMFP and DSEP functions for Mg, $\mathrm{Al}$, Si have been extracted from REELS in the framework of the two-layer model. To interpret Nb REELS spectra at several probing energies, we have tested a three-layer model with surface, intermediate and bulk layers. In this case, retrieval of DIIMFP and DSEP from Nb REELS spectra is performed in two steps. At the first step, DIIMFP of bulk, and DSEPs for surface and intermediate layers are extracted. At the second step, two upper layers are reduced into one surface layer with effective $\mathrm{DSEP}_{e}$. This model seems to be useful in practice since it simplifies the parametrization of DIIMFPs and their retrieval. The shape of the DIIMFP is given by a linear combination of basic functions (see Eqs. (17) and (18)), which do not change 


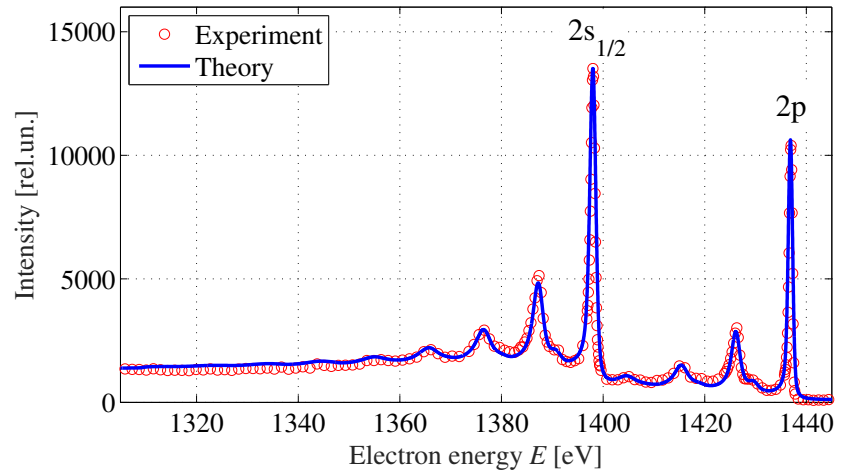

Figure 15: Comparison of the experimental PES spectrum [45] for Mg with that computed using obtained DIIMFPs from REELS. The average relative error is $7.8 \%$.

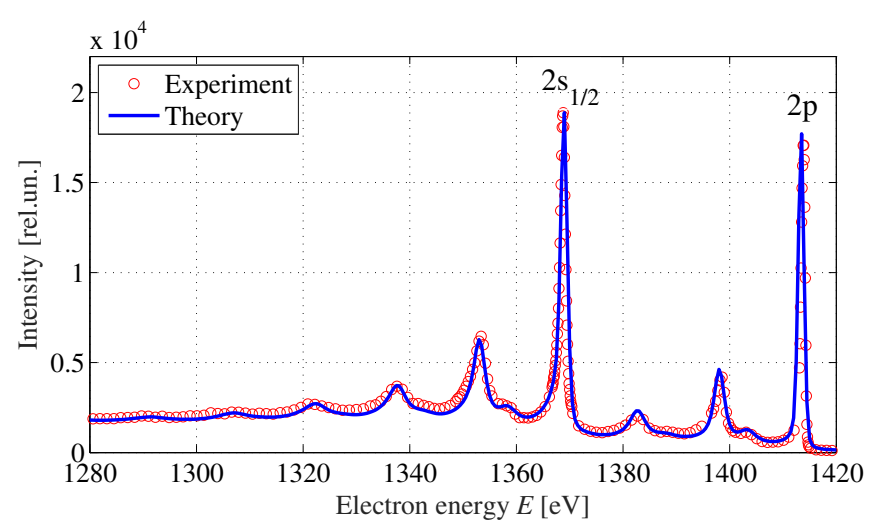

Figure 16: The same as Fig. 15 except for Al. The average relative error is $7.8 \%$.

with the initial energy.

We have applied the same retrieval strategy to PES spectra. The DIIMFP and DSEP for Be and W have been extracted. Finally, the DIIMFPs/DSEPs extracted from REELS have been validated by using them in PES computations. Good agreement is found between computed and measured spectra.

With the fast and flexible tool for REELS and PES spectra simulations, our intention is to consider larger sets of experimental data and to retrieve DIIMFP functions by using optimal estimation approach, wherein the residual is minimized over the whole available data. That will be the topic of our future papers.

\section{Acknowledgments}

The authors are grateful to two anonymous reviewers for their constructive comments, which helped us to improve the manuscript.

This work has been supported by the Russian Science Foundation under project No. 16-19-10027

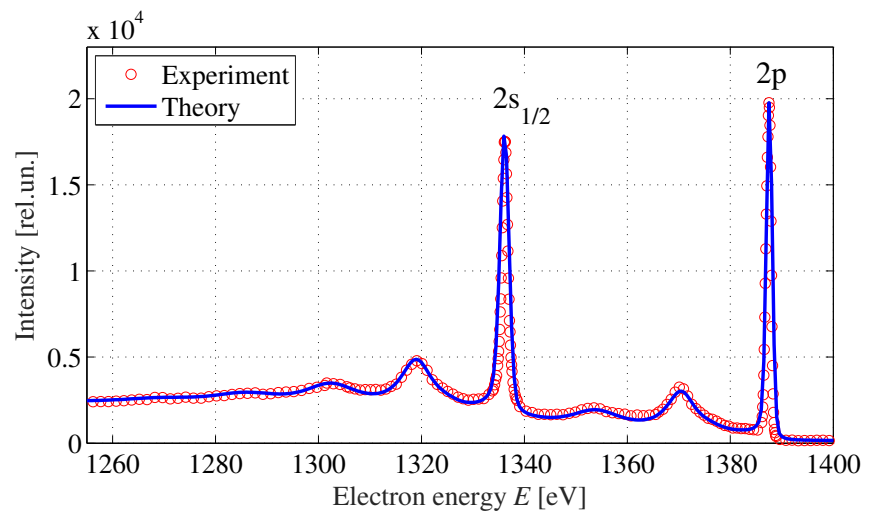

Figure 17: The same as Fig. 15 except for $\mathrm{Si}$. The average relative error is $4.3 \%$.

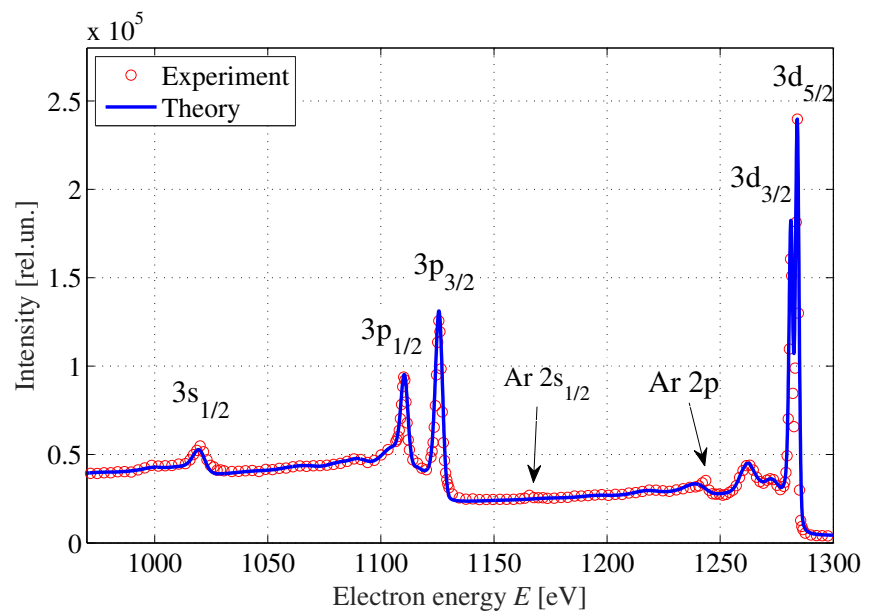

Figure 18: The same as Fig. 15 except for $\mathrm{Nb}$. The average relative error is $1.8 \%$.

\section{References}

[1] C. J. Powell, A. Jablonski, NIST Inelastic-Mean-Free-Path Database Version 1.2, SRD 71, National Institute of Standards and Technology, Gaithersburg, MD, 2010.

[2] L. Landau, E. Lifshitz, Theory of electromagnetism, Pergamom Press, Oxford, New York, 1977.

[3] K. Schwarz, P. Blaha, G. Madsen, Electronic structure calculations of solids using the WIEN2k package for material sciences, Computer Physics Communications 147 (2002) 71-76. doi:10.1016/ s0010-4655(02) 00206-0.

[4] E. Palik, Handbook of optical constnants of solids, Academic Press, New York, 1985.

[5] V. P. Afanas'ev, A. Lubenchenko, Energy spectra of reflected electrons at small energy losses, Surface Investigation X-Ray, Synchrotron and Neutron Techniques 13 (1998) 1087-1103.

[6] W. S. M. Werner, Electron transport in solids for quantitative surface analysis, Surface and Interface Analysis 31 (2001) 141-176. doi:10. 1002/sia. 973.

[7] S. Tougaard, I. Chorkendorff, Differential inelastic electron scattering cross sections from experimental reflection electron-energy-loss spectra: Application to background removal in electron spectroscopy, Physical Review B 35 (1987) 6570-6577. doi:10.1103/PhysRevB.35.6570.

[8] S. Tougaard, J. Kraaer, Inelastic-electron-scattering cross sections for $\mathrm{Si}$, 
$\mathrm{Cu}, \mathrm{Ag}, \mathrm{Au}, \mathrm{Ti}, \mathrm{Fe}$, and Pd, Physical Review B 43 (1991) 1651-1661. doi:10.1103/PhysRevB.43.1651.

[9] W. S. Werner, Differential probability for surface and volume electronic excitations in Fe, Pd and Pt, Surface Science 588 (2005) 26-40. doi:10. 1016/j.susc. 2005.05.023.

[10] W. S. Werner, M. R. Went, M. Vos, Surface plasmon excitation at a Au surface by $150-40,000 \mathrm{eV}$ electrons, Surface Science 601 (2007) L109L113. doi:10.1016/j.susc. 2007.06.076.

[11] V. P. Afanas'ev, D. S. Efremenko, a. V. Lubenchenko, Direct numerical reconstruction of inelastic cross sections from REELS and ISS spectra, Journal of Surface Investigation. X-ray, Synchrotron and Neutron Techniques 5 (2011) 375-382. doi:10.1134/S1027451011040033.

[12] W. S. Werner, Dielectric function of $\mathrm{Cu}, \mathrm{Ag}$, and $\mathrm{Au}$ obtained from reflection electron energy loss spectra, optical measurements, and density functional theory, Applied Physics Letters 89 (2006) 213106(1-3). doi:10.1063/1.2397026.

[13] P. C. Waterman, Matrix-exponential description of radiative transfer, J Opt Soc Am 71 (1981) 410-422. doi:10.1364/JOSA.71.000410.

[14] P. J. Flatau, G. L. Stephens, On the fundamental solution of the radiative transfer equation, J. Geophys. Res. 93 (1988) 11037. doi:10.1029/ jd093id09p11037.

[15] L. Landau, On the energy loss of fast particles by ionization, Journal of Physics-USSR 8 (1944) 201.

[16] W. S. M. Werner, Slowing down of medium-energy electrons in solids, Phys. Rev. B 55 (1997) 14925-14934. doi:10.1103/physrevb.55. 14925.

[17] V. Afanasyev, P. Kaplya, Consistent computations of electron energy losses in solids, Vestnik MEI 4 (2011) 90-96. [in Russian].

[18] V. Ambarzumian, Diffuse reflection of light by a foggy medium, Dokl. Akad. Nauk SSSR 38 (1943) 229-232. [in Russian].

[19] S. Chandrasekhar, Radiative Transfer, Dover Publications, Inc., New York, 1950.

[20] V. Afanas'ev, D. Efremenko, P. Kaplya, Analytical and numerical methods for computing electron partial intensities in the case of multilayer systems, Journal of Electron Spectroscopy and Related Phenomena 210 (2016) 16-29. doi:10.1016/j.elspec.2016.04.006.

[21] V. Afanas'ev, D. Efremenko, A. Lubenchenko, On the application of the invariant embedding method and the radiative transfer equation codes for surface state analysis, in: A. A. Kokhanovsky (Ed.), Light Scattering Reviews 8, Springer Praxis Books, Springer Berlin Heidelberg, Berlin, Heidelberg, 2013, pp. 363-423. doi:10.1007/978-3-642-32106-1_8.

[22] J. Peinado, J. Ibañez, V. Hernández, E. Arias, A family of BDF algorithms for solving Differential Matrix Riccati Equations using adaptive techniques, Procedia Computer Science 1 (2010) 2569-2577. doi:10 . 1016/j.procs. 2010.04.290.

[23] A. Doicu, T. Trautmann, Discrete-ordinate method with matrix exponential for a pseudo-spherical atmosphere: Scalar case, Journal of Quantitative Spectroscopy \& Radiative Transfer 110 (2009) 146-158. doi:10.1016/j.jqsrt.2008.09.014.

[24] D. Efremenko, A. Doicu, D. Loyola, T. Trautmann, Acceleration techniques for the discrete ordinate method, Journal of Quantitative Spectroscopy \& Radiative Transfer 114 (2013) 73-81. doi:10.1016/j . jqsrt.2012.08.014.

[25] D. Efremenko, D. Loyola, A. Doicu, R. Spurr, Multi-core-CPU and GPU-accelerated radiative transfer models based on the discrete ordinate method, Computer Physics Communications 185 (2014) 3079-3089. doi:10.1016/j.cpc.2014.07.018.

[26] V. Afanas'ev, P. Kaplya, A. Lubenchenko, O. Lubenchenko, Modern methods of transfer theory used for solution of signal identification problems of XPS, Vacuum 105 (2014) 96-101. doi:10.1016/j.vacuum. 2014.01 .010$.

[27] A. A. Radzig, B. M. Smirnov, Reference Data on Atoms, Molecules, and Ions, volume 31 of Springer Series in Chemical Physics,
Springer Berlin Heidelberg, Berlin, Heidelberg, 1985. doi:10.1007/ 978-3-642-82048-9.

[28] A. Jablonski, F. Salvat, C. J. Powell, NIST Electron Elastic-Scattering Cross-Section Database - Version 3.2, National Institute of Standards and Technology, Gaithersburg, MD, 2010.

[29] F. Salvat, A. Jablonski, C. J. Powell, ELSEPA - Dirac partial-wave calculation of elastic scattering of electrons and positrons by atoms, positive ions and molecules, Computer Physics Communications 165 (2005) 157190. doi:10.1016/j.cpc.2004.09.006.

[30] S. Tanuma, C. J. Powell, D. R. Penn, Calculations of electron inelastic mean free paths. VIII. Data for 15 elemental solids over the $50-2000 \mathrm{eV}$ range, Surface and Interface Analysis 37 (2005) 1-14. doi:10.1002/ sia.1997.

[31] S. Doniach, M. Sunjic, Many-electron singularity in X-ray photoemission and X-ray line spectra from metals, Journal of Physics C: Solid State Physics 3 (1970) 285-291. doi:10.1088/0022-3719/3/2/010.

[32] C. Biswas, A. Shukla, S. Banik, V. Ahire, S. Barman, Plasmons in corelevel photoemission spectra of Al(111), Phys. Rev. B 67 (2003). doi:10. $1103 /$ physrevb.67.165416.

[33] F. Yubero, S. Tougaard, Quantification of plasmon excitations in corelevel photoemission, Phys. Rev. B 71 (2005). doi:10.1103/physrevb. 71.045414.

[34] P. van Attekum, J. Trooster, Bulk- and surface-plasmon-loss intensities in photoelectron, Auger, and electron-energy-loss spectra of $\mathrm{Mg}$ metal, Physical Review B 20 (1979) 2335-2340. doi:10.1103/PhysRevB . 20 . 2335.

[35] N. Pauly, S. Tougaard, Model for Monte Carlo simulations of reflection electron energy loss spectra applied to Silicon at energies between 300 and $2000 \mathrm{eV}$, Surface and Interface Analysis 42 (2010) 1100-1104. doi:10.1002/sia.3277.

[36] E. A. Stern, R. A. Ferrell, Surface plasma oscillations of a degenerate electron gas, Phys. Rev. 120 (1960) 130-136. doi:10.1103/PhysRev . 120.130

[37] W. Werner, http://eaps4.iap.tuwien.ac.at/ werner, Accessed: 2015-09-30

[38] W. Werner, Differential surface and volume excitation probability of medium-energy electrons in solids, Physical Review B 74 (2006) 075421. doi:10.1103/PhysRevB.74.075421.

[39] M. Vos, G. P. Cornish, E. Weigold, High-energy (e, 2e) spectrometer for the study of the spectral momentum density of materials, Review of Scientific Instruments 71 (2000) 3831. doi:10.1063/1.1290507.

[40] V. P. Afanasyev, D. S. Efremenko, A. V. Lubenchenko, M. Vos, M. R. Went, Extraction of cross-sections of inelastic scattering from energy spectra of reflected atomic particles, Bulletin of the Russian Academy of Sciences: Physics 74 (2010) 170-174. doi:10.3103/ S1062873810020152.

[41] Y. Chen, Surface effects on angular distributions in X-ray-photoelectron spectroscopy, Surface Science 519 (2002) 115-124. doi:10.1016/ s0039-6028 (02) 02206-9.

[42] V. P. Afanas'ev, A. V. Lubenchenko, M. V. Lukashevsky, M. Norell, A. B. Pavolotsky, Study of $\mathrm{Al} / \mathrm{Nb}$ interface by spectroscopy of reflected electrons, Journal of Applied Physics 101 (2007) 064912. doi:10.1063/1. 2716385.

[43] M. Trzhaskovskaya, V. Nefedov, V. Yarzhemsky, Photoelectron angular distribution parameters for elements $Z=1$ to $Z=54$ in the photoelectron energy range RANGE 100-5000 eV, Atomic Data and Nuclear Data Tables 77 (2001) 97-159. doi:10.1006/adnd. 2000.0849.

[44] M. Trzhaskovskaya, V. Nefedov, V. Yarzhemsky, Photoelectron angular distribution parameters for elements $\mathrm{Z}=55$ to $\mathrm{Z}=100$ in the photoelectron energy range $100-5000 \mathrm{eV}$, Atomic Data and Nuclear Data Tables 82 (2002) 257-311. doi:10.1006/adnd.2002.0886

[45] J. Moulder, W. Stickle, P. Sobol, K. Bomben, Handbook of X Ray Photoelectron Spectroscopy, Physical Electronics, 1995. 